Check for updates

Cite this: RSC Adv., 2019, 9, 29772

Received 2nd June 2019

Accepted 23rd August 2019

DOI: $10.1039 / c 9 r a 04166 d$

rsc.li/rsc-advances

\title{
Renewable 4-HIF/NaOH aerogel for efficient methylene blue removal via cation $-\pi$ interaction induced electrostatic interaction $\uparrow$
}

\author{
Longfei Zhang, Li Yang, (D) * Yewei Xu and Guanjun Chang (iD* \\ A novel porous organic material 4-hydroxyindole-formaldehyde/ $\mathrm{NaOH}(4-\mathrm{HIF} / \mathrm{NaOH})$ aerogel was \\ prepared via a facile polymerization, soaking in $\mathrm{NaOH}$ aqueous solution and ambient drying method. 4- \\ $\mathrm{HIF} / \mathrm{NaOH}$ aerogel porous polymer networks with high surface area have been applied as efficient \\ adsorbents to remove methylene blue from wastewater via synergistic effects of cation $-\pi$ interaction \\ induced electrostatic interaction, electrostatic interaction and $\pi-\pi$ interaction. The adsorption capacity \\ calculated by adsorption isotherms at $303 \mathrm{~K}$ was $1016.9 \mathrm{mg} \mathrm{g}^{-1}$ which is higher than those observed for \\ methylene blue on other aerogels and most other materials. Furthermore, the methylene blue loaded 4- \\ HIF aerogel can easily be regenerated with $0.1 \mathrm{M} \mathrm{HCl}$ solution and ethanol wash, retaining over $75 \%$ of \\ the adsorption capacity after recycling five times.
}

\section{Introduction}

Commercially dyes are widely used in many industries, such as textiles, paper, plastics, printing, tannery and paints. ${ }^{1,2}$ When those synthetic dyes are released into natural water bodies, they seriously affect aquatic life, as well as the food chain, and pose harmful effects to human health, including teratogenetic, carcinogenic and mutagenic effects..$^{3-5}$ Many treatment methods for removing dyes from wastewater have been employed, including photochemical oxidation, membrane separation, biodegradation and adsorption. ${ }^{6-8}$ Adsorption has been considered to be superior to other technologies in terms of high efficiency, low cost, easy operation, and insensitivity to toxic substrates. ${ }^{9}$

Numerous adsorbents have been reported including clay minerals, agricultural solid wastes, graphene oxide nanofibrous composite membrane and activated carbon..$^{10,11}$ However, most of the adsorbents suffer from either limited adsorption capacities or unsatisfactory drawbacks in terms of operation and recycling. For example, the graphene-based materials have attracted tremendous interest for their valuable properties such as abundant oxygen-containing groups and large specific surface. The main shortcomings of graphene are easy agglomeration during storage, which results in the decline of adsorption capacity in practical application. ${ }^{12}$ The frequently-used

State Key Laboratory of Environment-friendly Energy Materials, School of Material Science and Engineering, Southwest University of Science and Technology, Mianyang, 621010, P. R. China.E-mail: gjchang@mail.ustc.edu.cn

$\dagger$ Electronic supplementary information (ESI) available: BET analysis of alkaline $4-\mathrm{HIF} / \mathrm{NaOH}$ aerogel; pore size distribution curve; effects of initial $\mathrm{pH}$ on the adsorption and date. See DOI: 10.1039/c9ra04166d activated carbon with great adsorption capacity is costly in operation and regeneration and has poor dye specificity. ${ }^{13,14}$ Páez reported a $\mathrm{CO}_{2}$ activated resorcinol/formaldehyde carbon xerogels to adsorb methylene blue (MB), the process was high cost and time consuming and the regeneration experiments was not mentioned..$^{15} \mathrm{Yu}$ et al. obtained a graphene oxide/chitosan aerogel microspheres as adsorbent for broad spectrum and rapid adsorption of water contaminants, however, the synthetic routes posed challenge in operation. ${ }^{16}$ Thus, development of recyclable adsorbent with facile operation and high adsorption capacity to capture MB from wastewater was a challenging and meaningful task.

The cation- $\pi$ interaction describes the association between a cation and the face of a molecule containing a $\pi$ system, which is the strongest non-covalent interaction as known. ${ }^{17-19}$ As an electron-rich $\pi$ system, indole is an excellent platform for cation $-\pi$ interaction. Hydroxyl group as electron-donating substituent at 4-position affording increasingly negative electrostatic potentials above and below the ring plane, predicting a stronger interaction with a cation. ${ }^{20}$ Metal cation- $\pi$ interactions are well known, ${ }^{21}$ Reddy and co-workers have systematically studied the cation- $\pi$ interactions between alkali/alkaline earth metals and indole. ${ }^{22}$

Besides the longstanding studies in indole-based supramolecular materials of our group ${ }^{23-26}$ and enlightened by the studies of Juszczak's research group ${ }^{27}$ in basic condition, cation ions (i.e. $\mathrm{Na}^{+}$) are available for binding indole via cation- $\pi$ interactions, in which $\mathrm{Na}^{+}$act as electron acceptors and indole serve as electron donors. Highest occupied molecular orbital (HOMO) $\pi$-electron density is delocalized towards the $\mathrm{Na}^{+}$, creating a partial electron deficiency on the indole ring. Meanwhile, hydrogen bond was formed at indole amine 
hydrogen with hydroxyl anion, which creating a compensatory, stabilizing partial negative charge on the indole. In this way, the $\mathrm{Na}^{+}$-indole- $\mathrm{OH}^{-}$complex could be established stable and more interestingly, the negative charged active sites were exposed to aqueous environment, which renders the electrostatic attraction of cationic dye happened efficiently and this process was named cation $-\pi$ induced electrostatic interaction (CIE).

On the other hand, the $\mathrm{p} K_{\mathrm{a}}$ for hydroxyl group of 4-hydroxyindole is $\sim 10.68,{ }^{28}$ which would be deprotonated to form -Ogroups in basic solution. The -O- groups could also capture cationic dye via electrostatic interactions, which is another important contributory factor that facilitate cationic pollutants collection. Furthermore, we choose MB as a model compound for cationic dyes, which is an ideally planar molecule with aromatic backbone and 4-HIF aerogel network also contain abundant aromatic rings, the $\pi-\pi$ interactions could occur between MB molecules and 4-HIF aerogel network. ${ }^{29}$

Inspired by this interesting study, we hypothesized that the MB can be efficiently removed from contaminated water by involving both hydroxyl group and negatively charged $\mathrm{OH}^{-}$ induced by indole groups in porous materials where synergistic effects of CIE, electrostatic interaction and $\pi-\pi$ interactions work for MB capture. Bearing these in mind, in this paper, we designed and synthesized 4-HIF aerogel by facile polymerization followed by ambient drying method. Then, highly in accordance with our design strategy, large amounts of MB left in wastewater were able to be collected and concentrated by 4-HIF aerogel successfully. Moreover, the adsorption isotherm, kinetic, and thermodynamic characteristics as well as the mechanism were fully studied to gain a deeper insight into adsorption process.

\section{Experimental}

\section{Main materials and measurements}

Methylene blue $\left(\mathrm{C}_{16} \mathrm{H}_{18} \mathrm{ClN}_{3} \mathrm{~S}\right.$, MW: 319.9) and 4-hydroxyindole was purchased from aladdin Chemical Reagent Co., Ltd. Methyl orange, formaldehyde (37\%) were supplied by Kelong Chemical Reagent Factory (Chengdu, China). Manganese sulfate monohydrate $\left(\mathrm{MnSO}_{4} \cdot \mathrm{H}_{2} \mathrm{O}\right)$, silver nitrate $\left(\mathrm{AgNO}_{3}\right)$, lead nitrate $\left(\mathrm{Pb}\left(\mathrm{NO}_{3}\right)_{2}\right)$, (cobaltous chloride $\left.\left(\mathrm{CoCl}_{2}\right)\right)$ and mercury chloride $\left(\mathrm{HgCl}_{2}\right)$ were also supplied by Aladdin. Acetone and sodium hydroxide $(\mathrm{NaOH})$ were of analytical grade. All reagents were purchased from commercial suppliers and were used without further purification.

FT-IR spectra were recorded on a Nicolet 6700 FTIR spectrometer in the region of $4000-400 \mathrm{~cm}^{-1}$ using $\mathrm{KBr}$ pellets. The scanning electron microscopy (SEM) images of the 4-HIF aerogel, were made on an Ultra 55 microscope system. Surface areas and pore size distributions were measured by nitrogen $\left(\mathrm{N}_{2}\right)$ adsorption and desorption at 77.3 K on a Quantachrome automated adsorption analyzer. Specific surface areas were calculated according to the Brunauer-Emmett-Teller (BET) method. Solid-state cross-polarization magic-angle-spinning (CP/MAS) NMR spectrum was recorded on a Bruker Advance III 400 NMR spectrometer. The zeta potential of 4-HIF aerogel was measured by a Zetasizer nano potential analyzer (Zeta PAL). The metal ion concentrations in solutions before and after adsorption were measured using ICP-AES (Jarrell-ASH, ICAP-9000). The concentrations of dyes were obtained by a Shimadzu UV-3150 spectrophotometer.

\section{Preparation of 4-HIF aerogel}

The synthesis process of 4-HIF aerogel was depicted in Fig. 1. Specifically, to an ampoule bottle equipped with 4-hydroxyindole $(0.2663 \mathrm{~g}, 2 \mathrm{mmol}), \mathrm{Na}_{2} \mathrm{CO}_{3}(0.2120 \mathrm{~g}, 2 \mathrm{mmol})$ and deionized water $(1.75 \mathrm{~mL})$ at room temperature and the above mixture was stirred at $70{ }^{\circ} \mathrm{C}$ for $2 \mathrm{~min}$. Then a solution of $37 \%$ formaldehyde $(0.3 \mathrm{~mL})$ was added to afford a weak gel and kept for $10 \mathrm{~h}$ at $70{ }^{\circ} \mathrm{C}$ in an oil bath to yield a robust gel. Upon removal from ampoule bottle, the gel was placed in a $2 \%$ acetic acid solution at $45{ }^{\circ} \mathrm{C}$ for $48 \mathrm{~h}$ to increase the cross-link density through further condensation of hydroxymethyl groups and the excess $\mathrm{Na}_{2} \mathrm{CO}_{3}$ were neutralized simultaneously. Then the obtained 4-HIF hydrogel was washed by deionized water and soaked in acetone for $48 \mathrm{~h}$ successively followed by ambient drying at $60{ }^{\circ} \mathrm{C}$ to form the neutral 4-HIF aerogel. The synthesis process of alkaline 4-HIF aerogel was the same as neutral ones except for immerging the obtained 4-HIF hydrogel in $\mathrm{NaOH}$ aqueous solution $(0.5 \mathrm{M}, 25 \mathrm{~mL})$ for $12 \mathrm{~h}$ before solvent exchange in acetone.

\section{Dye adsorption and regeneration}

All adsorption experiments were carried out by using alkaline 4HIF aerogel unless otherwise stated. To investigate the effect of $\mathrm{pH}$ on adsorption for $\mathrm{MB}$, the $\mathrm{pH}$ of $\mathrm{MB}$ solution $\left(350 \mathrm{mg} \mathrm{L}^{-1}\right)$ was adjusted by $1.0 \mathrm{~mol} \mathrm{~L}^{-1} \mathrm{NaOH}$ and $1.0 \mathrm{~mol} \mathrm{~L}^{-1} \mathrm{HCl}$ aqueous solution. Adsorption isotherm experiment was conducted at 303 $\mathrm{K}$ by adding $10 \mathrm{mg}$ 4-HIF aerogel onto $40 \mathrm{~mL}$ solution with $\mathrm{MB}$ concentration ranging from 140 to $380 \mathrm{mg} \mathrm{L}^{-1}$. Adsorption kinetic experiment were performed by mixing 4-HIF aerogel (50 $\mathrm{mg}$ ), MB solution $\left(200 \mathrm{~mL}, 300 \mathrm{mg} \mathrm{L}^{-1}\right)$ followed by stirring at $303 \mathrm{~K}$ for $24 \mathrm{~h}$. Adsorption thermodynamics experiments were carried out adding $10 \mathrm{mg}$ 4-HIF aerogel onto $40 \mathrm{~mL} \mathrm{MB}$ solution

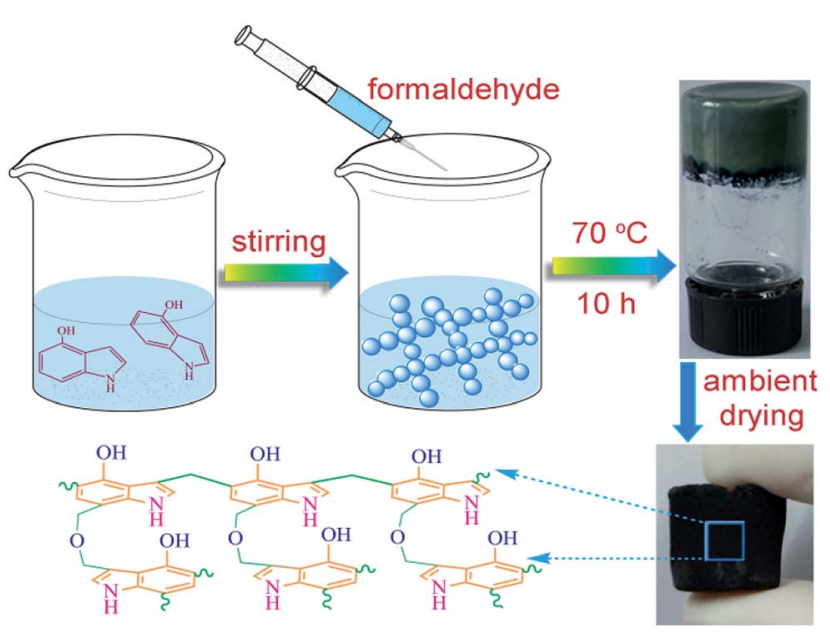

Fig. 1 Synthetic scheme of neutral 4-HIF aerogel. 
(300 $\mathrm{mg} \mathrm{L}^{-1}$ ) and stirred at $303 \mathrm{~K}, 313 \mathrm{~K}, 323 \mathrm{~K}$ respectively. After adsorption, the 4-HIF aerogels were separated from solutions using $0.45 \mu \mathrm{m}$ polypropylene syringe filters. The absorbance of MB was detected by a UV-vis spectrophotometer, and the concentration was calculated by comparing the appropriate calibration curve. The adsorption capacity $\left(q_{t}\right.$, dye removal per gram of 4-HIF aerogel at time $t, \mathrm{mg}^{-1}$ ) and $q_{\mathrm{e}}$ (dye adsorbed per gram of 4-HIF aerogel at equilibrium $\left.\left(\mathrm{mg} \mathrm{g}^{-1}\right)\right)$ were calculated according to the following equations:

$$
\begin{gathered}
q_{t}=\frac{\left(C_{0}-C_{t}\right) V}{m} \\
q_{\mathrm{e}}=\frac{\left(C_{0}-C_{\mathrm{e}}\right) V}{m}
\end{gathered}
$$

where $C_{\mathrm{e}}\left(\mathrm{mg} \mathrm{L}^{-1}\right)$ represents the concentration of dye in aqueous solution at equilibrium, $m(\mathrm{~g})$ is the mass of 4-HIF aerogel, and $V(\mathrm{~L})$ is the volume of dye solution. The removal efficiency of dye was calculated by the following equation:

$$
E(\%)=\frac{C_{0}-C_{\mathrm{e}}}{C_{0}} \times 100 .
$$

The adsorption experiment was carried out in duplicate, and the experimental point was selected the mean of duplicates. For desorption experiment, after saturation, the mixture was filtered, and the residual 4-HIF aerogel was processed with absolute ethanol at $50{ }^{\circ} \mathrm{C}$ and stirred for $5 \mathrm{~min}$. Besides, $0.1 \mathrm{M}$ $\mathrm{HCl}$ was also added to improve the regeneration efficiency. The adsorbent was separated and then successively treated by $50 \mathrm{~mL}$ deionized water and $0.5 \mathrm{M} \mathrm{NaOH}$ again followed by filtration and ambient drying to reused for adsorption.

\section{Real water sample analysis}

River water samples were collected from Fujiang River (Mianyang, China). The obtained water samples were filtered and diluted 100 times with ultrapure water and were spiked with heavy metal ions $\left(\mathrm{Pb}^{2+}, \mathrm{Ag}^{+}, \mathrm{Hg}^{2+}, \mathrm{Co}^{2+}, \mathrm{Mn}^{2+}\right)$ and dyes (MO, $\mathrm{MB})$ with a concentration of $30 \mathrm{mg} \mathrm{L}^{-1}$ and $260 \mathrm{mg} \mathrm{L}^{-1}$ respectively. The adsorption experiments were carried out at natural $\mathrm{pH}$ values which ranged from 5.8 to 6.2 at room temperature with the dosage of $0.25 \mathrm{~g} \mathrm{~L}^{-1}$. The concentrations of heavy metal ions and dyes in the samples before and after adsorption were determined by ICP-AES and UV-vis spectrometry, respectively.

\section{Results and discussion}

\section{Characterization of 4-HIF aerogel}

To identify the structure of 4-HIF aerogel, FT-IR spectrum was investigated and shown in Fig. 2a. The strong peak at $3404 \mathrm{~cm}^{-1}$ was attributed to the overlap of $\mathrm{O}-\mathrm{H}$ and $\mathrm{N}-\mathrm{H}$ stretching vibration in 4-hydroxyindole. ${ }^{30,31}$ The peak at $2923 \mathrm{~cm}^{-1}$ was assigned to the $-\mathrm{CH}_{2}-$ units, ${ }^{32}$ and the band at $1244 \mathrm{~cm}^{-1}$ was associated with $\mathrm{CH}_{2}-\mathrm{O}-\mathrm{CH}_{2}$ stretching vibrations of methylene ether bridges, ${ }^{33}$ indicating that polycondensation of formaldehyde and 4-hydroxyindole successfully compounded into 4-HIF
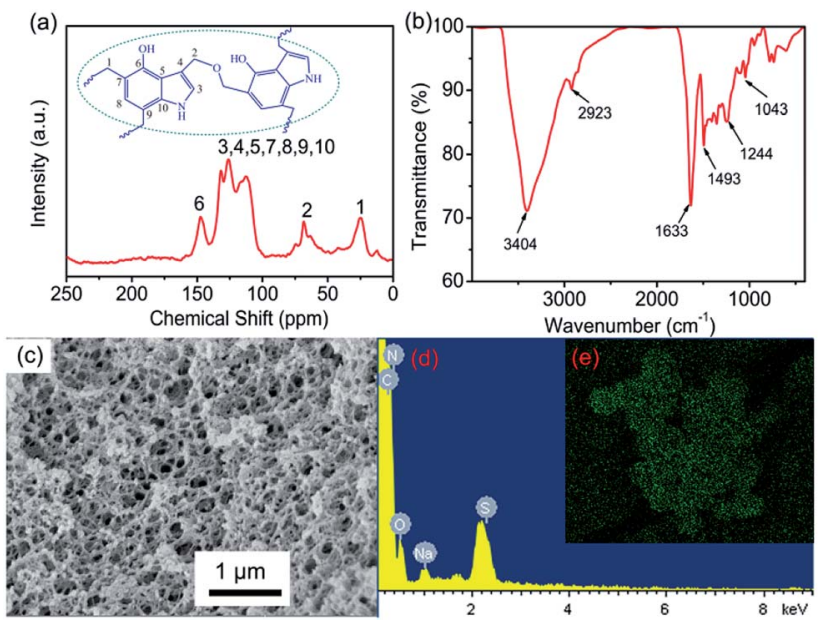

Fig. 2 (a) Solid-state ${ }^{13} \mathrm{C}$ NMR of neutral 4-HIF aerogel; (b) FT-IR spectrum of neutral 4-HIF aerogel; (c) SEM image of alkaline 4-HIF aerogel; (d) EDS of 4-HIF alkaline aerogel after adsorbing MB; (e) elemental mapping of $\mathrm{S}$.

aerogel. The result was also confirmed by solid-state ${ }^{13} \mathrm{C}$ NMR (Fig. 2b), the broad peaks in NMR spectrum at $150-110 \mathrm{ppm}$ were ascribed to the indole ring carbons, and the peaks located at 75-25 ppm corresponded to the methylene carbons. This finding was further supported that 4-HIF aerogel network structure was formed.

The microstructure of alkaline 4-HIF aerogel was investigated by SEM. As shown in Fig. 2c, the 3D network was well established in 4-HIF aerogel, 4-hydroxyindole fabricated the highly crosslinked network with formaldehyde and the structure looks like a three-dimensional "web". $\mathrm{N}_{2}$ adsorptiondesorption analysis confirmed the high specific surface area $\left(130 \mathrm{~m}^{2} \mathrm{~g}^{-1}\right)$ of 4 -HIF aerogel. The pore size distribution curve showed the existence of a mass of meso- and macropores and the average pore diameter was $46 \mathrm{~nm}$ (Fig. S1 $\dagger$ ). Obviously, the high specific surface area and a large amount of pores endow 4HIF aerogel with easy and quick mass penetration during adsorption.

\section{Dye adsorption}

To evaluate the performance of 4-HIF aerogel for $\mathrm{MB}$, the adsorption experiments were carried out by adding $10 \mathrm{mg}$ 4-HIF aerogel into $40 \mathrm{~mL}$ (adsorbent dosage $=0.25 \mathrm{~g} \mathrm{~L}^{-1}$ ) aqueous solution containing an initial MB concentration of $260 \mathrm{mg} \mathrm{L}^{-1}$ at $303 \mathrm{~K}$. After stirred for $12 \mathrm{~h}$, the adsorption equilibrium achieved and the color of MB solution changed from dark blue to light blue and absorbance intensity of MB decrease rapidly. The adsorption capacity at adsorption equilibrium was up to $961.4 \mathrm{mg} \mathrm{g}^{-1}$ and the removal efficiency of MB was as high as 92.4\%. Energy dispersive X-ray spectroscopy (EDS) measurements and elemental mapping verified the existence of $\mathrm{S}$ in the 4-HIF aerogel after adsorbing MB (Fig. 2d and e) indicating MB was indeed captured into the aerogel. Besides, MB adsorption experiment employed the neutral 4-HIF aerogel was also carried out at the same condition. The $q_{\mathrm{e}}$ was only $104.6 \mathrm{mg} \mathrm{g}^{-1}$, which 
very close to the $q_{\mathrm{e}}\left(98.82 \mathrm{mg} \mathrm{g}^{-1}\right)$ reported by $\mathrm{Fu}$ and co-workers who employed polydopamine (PDA) microspheres consisted of 5,6-dihydroxyindole unit. ${ }^{29}$

In addition, polypropylene syringe filters $(0.45 \mu \mathrm{m})$ equipped with 4-HIF aerogel powder and syringe $(5 \mathrm{~mL})$ were used to dye adsorption in miniature-scale water purification, as shown in Fig. 3. The solution with $\mathrm{MB}$ concentration of $50 \mathrm{mg} \mathrm{L}^{-1}$ was filtered through continuous filtration. After $30 \mathrm{~s}$, about $4 \mathrm{~mL}$ solution was filtered through 4-HIF aerogel, and colorless pure water was collected, indicating that the 4-HIF aerogel could rapidly removed the $\mathrm{MB}$. The characteristic UV absorption peaks of MB disappeared (Fig. 3c), indicating complete removal of MB from solution.

\section{Effects of initial solution pH}

The effects of initial solution $\mathrm{pH}$ on MB adsorption onto 4-HIF aerogel were studied within the $\mathrm{pH}$ range between 2 and 10 at room temperature, as shown in Fig. $\mathrm{S} 4 . \dagger$ As the solution $\mathrm{pH}$ decreased from 7 to 2 , the adsorption capacity underwent a distinct decrease. This observed behavior may be explained by the charge of adsorbent and molecular charge of adsorbate. With pH decreased, more hydroxide anion would be neutralized resulting in the broken of hydrogen bonds, which interrupted the CIE interaction. Moreover, $\mathrm{MB}$ is a cationic dye and contains positively charged quaternary ammonium group $\left(\mathrm{N}^{+}\left(\mathrm{CH}_{3}\right)_{2} \mathrm{Cl}^{-}\right)$ at acidic condition, ${ }^{34}$ the high $\mathrm{H}^{+}$concentration would compete strongly with MB cations for the available adsorption sites. The -O- groups of the aerogel would be neutralized and protonated to form $-\mathrm{OH}^{2+}$ which would lead to electrostatic repulsion with MB cations to decrease the adsorption capacity. In extremely acid condition, electrostatic interaction become less significant
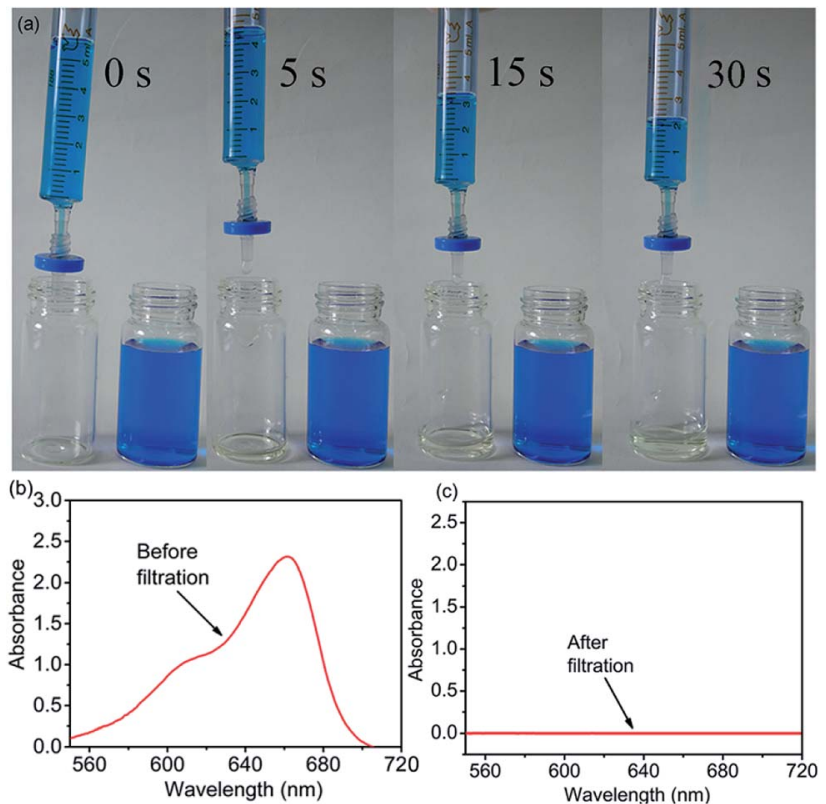

Fig. 3 (a) Images of the apparatus used for continuous filtration and the solution before and after filtration (4-HIF aerogel powder was stuck in $0.45 \mu \mathrm{m}$ polypropylene syringe filter). UV absorption spectra of the solution before (b) and after (c) filtration. and $\pi-\pi$ interactions act as the main drying force between the adsorbent and adsorbate. ${ }^{29}$ By contrast, the adsorption capacities of MB present a significant increasing trend from 938.6 to $1284.1 \mathrm{mg} \mathrm{g}^{-1}$ as the $\mathrm{pH}$ increased from 7 to 10 . And the $\mathrm{MB}$ removal efficiency can reach up to $91.7 \%$ when the initial $\mathrm{pH}$ of MB solution is 10 . With the $\mathrm{pH}$ increasing, the more hydroxyl groups of the aerogel would be deprotonated to form -Ogroups and the $\mathrm{Na}^{+}$-indole- $\mathrm{OH}^{-}$complex was formed stable, thus the active sites density increased and the electrostatic attraction were enhanced. It suggested 4-HIF aerogel can be a high-efficiency adsorbent for the adsorption of MB and basic solutions benefit MB adsorption.

\section{Adsorption kinetic studies}

Dye adsorption rates of 4-HIF aerogel was measured. It can be seen from Fig. 4a, $q_{t}$ increased rapidly at the initial $20 \mathrm{~min}$ and adsorption capacity was up to $614.3 \mathrm{mg} \mathrm{g}^{-1}$. After $2 \mathrm{~h}, 83.6 \%$ of equilibrium adsorption capacity was reached and $q_{t}$ increased slowly to equilibrium at $8 \mathrm{~h}$. Then, the pseudo-first-order (eqn (4)) and pseudo-second-order (eqn (5)) models were applied to quantitatively analyze the adsorption kinetic curve (Fig. 4b).

$$
\begin{gathered}
\log \left(q_{\mathrm{e}}-q_{t}\right)=\log q_{\mathrm{e}}-\frac{K_{1} t}{2.303} \\
\frac{1}{q_{t}}=\frac{1}{K_{2} q_{\mathrm{e}}^{2}}+\frac{t}{q_{\mathrm{e}}}
\end{gathered}
$$

where $q_{\mathrm{e}}$ and $q_{t}$ are the amount of adsorbed dye $\left(\mathrm{mg} \mathrm{g}^{-1}\right)$ at equilibrium and at time $t(\mathrm{~min}), K_{1}\left(\mathrm{~min}^{-1}\right)$ and $K_{2}(\mathrm{~g}$ $\mathrm{mg}^{-1} \mathrm{~min}^{-1}$ ) are the rate constants of the pseudo-first-order and pseudo-second-order, respectively.

The pseudo-first-order model assumes that the internal diffusion process is the rate-controlling step, whereas the pseudo-second-order kinetic model considers that the chemisorption of the adsorbate molecules onto the active adsorption sites is the rate-controlling step. $^{\mathbf{1 6}}$ Comparing the two
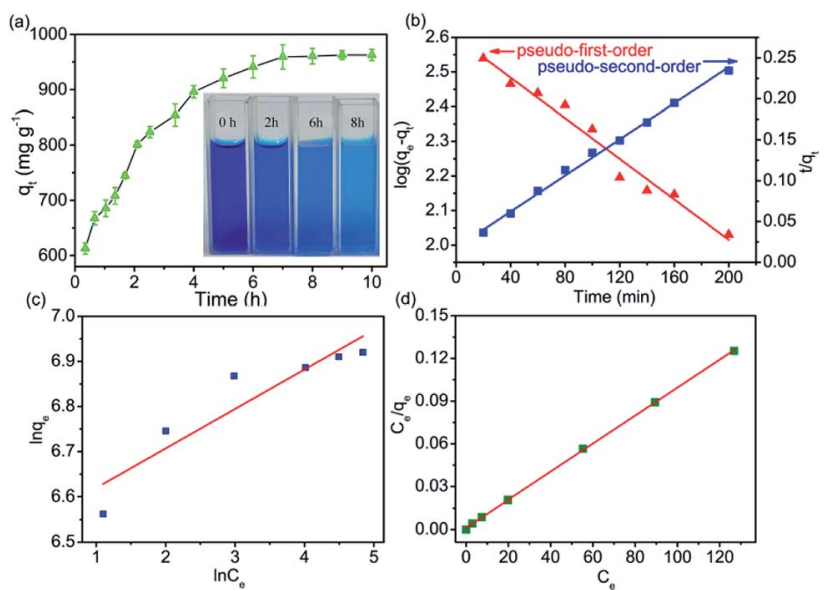

Fig. 4 (a) Adsorption kinetic of MB on 4-HIF aerogel. Inset: the photographs of color change of MB. (b) Fitting results of MB on 4-HIF aerogel for kinetic models. (c) Freundlich isotherm and (d) Langmuir adsorption isotherm plots for the adsorption of MB by 4-HIF aerogel. 
correlation coefficients $\left(R^{2}\right)$ displayed in Table S3, $\dagger$ the pseudosecond-order kinetic model $\left(R^{2}=0.994\right)$ had a better correlation than the pseudo-first-order kinetic model $\left(R^{2}=0.966\right)$ for adsorption of MB with 4-HIF aerogel. It was clear that the adsorption process of $\mathrm{MB}^{+}$was mainly ruled by the chemisorption between MB and active sites of 4-HIF aerogel.

\section{Adsorption isotherm}

Adsorption isotherm models were determined by fitting the experimental data to Langmuir and Freundlich isotherm models. The linearized Langmuir isotherm eqn (6) and Freundlich isotherm eqn (7) can be expressed as follows:

$$
\begin{gathered}
\frac{C_{\mathrm{e}}}{q_{\mathrm{e}}}=\frac{C_{\mathrm{e}}}{q_{\mathrm{m}}}+\frac{1}{q_{\mathrm{m}} K_{\mathrm{L}}} \\
\ln q_{\mathrm{e}}=\ln K_{\mathrm{f}}+\frac{1}{n} \ln C_{\mathrm{e}}
\end{gathered}
$$

where $C_{\mathrm{e}}$ is the concentration $\left(\mathrm{mg} \mathrm{L}^{-1}\right)$ of the solution at equilibrium; $q_{\mathrm{e}}$ is the equilibrium adsorption capacity $\left(\mathrm{mg} \mathrm{g}^{-1}\right) ; q_{\mathrm{m}}$ is the maximum adsorption capacity $\left(\mathrm{mg} \mathrm{g}^{-1}\right) ; K_{\mathrm{L}}$ is the Langmuir isothermal constant $\left(\mathrm{L} \mathrm{mg}^{-1}\right) ; K_{\mathrm{f}}$ is the Freundlich isothermal constant $\left(\mathrm{mg} \mathrm{g}^{-1}\right)(\mathrm{L} \mathrm{mg})^{1 / n}$; and $n$ is the factor relating to the adsorption intensity.

The fitting results of the adsorption isotherm models were displayed in Fig. $4 \mathrm{c}$ and d. According to the $R^{2}$ presented in Table S4, $\uparrow$ the Langmuir model $\left(R^{2}=0.999\right)$ was much higher than Freundlich model $\left(R^{2}=0.833\right)$, indicating that the Langmuir equation could fit the experimental adsorption data better. The calculated adsorption capacity was $1016.9 \mathrm{mg} \mathrm{g}^{-1}$, which was higher than other aerogels and most other materials (as shown in Table 1). Especially, this adsorption capacity was much better than that of walnut shell-based activated carbon $\left(315 \mathrm{mg} \mathrm{g}^{-1}\right)^{36}$ and graphene oxide composite aerogel $(578 \mathrm{mg}$ $\left.\mathrm{g}^{-1}\right),,^{34}$ indicating that 4 -HIF aerogel was a competitive candidate for wastewater purification.

\section{Adsorption thermodynamics}

To determine the adsorption nature, the adsorption thermodynamics were studied. The effect of temperature on the sorption of MB onto 4-HIF aerogel at $303 \mathrm{~K}, 313 \mathrm{~K}$ and $323 \mathrm{~K}$ with an initial concentration of $300 \mathrm{mg} \mathrm{L^{-1 }}$ were investigated. The enthalpies were calculated by Van't Hoff equations. ${ }^{38}$ The enthalpy change $(\Delta H)$ and entropy change $(\Delta S)$ can be calculated from the equation:

$$
\begin{aligned}
& \ln \frac{q_{\mathrm{e}}}{C_{\mathrm{e}}}=-\frac{\Delta H}{R T}+\frac{\Delta S}{R} \\
& \Delta G=\Delta H-T \Delta S
\end{aligned}
$$

where $q_{\mathrm{e}}\left(\mathrm{mg} \mathrm{g}^{-1}\right)$ is the adsorption capacity, $C_{\mathrm{e}}\left(\mathrm{mg} \mathrm{L}^{-1}\right)$ is the MB concentrations at equilibrium, $T$ is absolute temperature in Kelvin (K), $R$ is the universal gas constant $\left(8.314 \mathrm{~J} \mathrm{~mol}^{-1} \mathrm{~K}^{-1}\right)$. The thermodynamic parameters were calculated and listed in Table S5. $\dagger$ The $\Delta G$ values were negative at all the studied temperatures, indicating the spontaneous adsorption nature for MB. Importantly, the positive $\Delta H$ value for MB indicated that MB sorption onto 4-HIF aerogel was an endothermic process and the adsorption capacities of MB presented a significant increasing trend from 968.8 to $1075.4 \mathrm{mg} \mathrm{g}^{-1}$ with the increase of temperature values in the range of $303 \mathrm{~K}$ to $323 \mathrm{~K}$.

\section{Adsorption mechanism}

The schematic process and adsorption mechanisms were shown in Fig. 5 and summarized as follow. (i) According to the adsorption results, compared with the neutral 4-HIF aerogel, more than 8 -fold improvement in $q_{\mathrm{e}}$ was observed by using alkaline ones, indicating pretreatment by $\mathrm{NaOH}$ before drying was a critical process resulting in the prominent performance. As mentioned above, $\mathrm{Na}^{+}$could combined with indole through cation $-\pi$ interaction, hydroxide anion hydrogen bonds to the indole amine, so the $\mathrm{Na}^{+}$-indole- $\mathrm{OH}^{-}$complex was formed stably. Then, the negatively charged $\mathrm{OH}^{-}$could act as active sites to adsorb dye molecules through CIE interaction. FT-IR spectra of 4-HIF aerogel before and after MB adsorption were compared in Fig. S7. $\dagger$ The broad band at $3422 \mathrm{~cm}^{-1}$ before sorption, assigned to $\mathrm{N}-\mathrm{H}$ stretching vibrations, shifted to $3403 \mathrm{~cm}^{-1}$ after adsorption and the peak at $1632 \mathrm{~cm}^{-1}$ assigned to $\mathrm{N}-\mathrm{H}$ bending vibration shifted to $1596 \mathrm{~cm}^{-1}$ overlapped with the aromatic rings vibration of $\mathrm{MB},{ }^{37,39}$ suggesting participation of the hydroxide anions in the adsorption process. (ii) Deprotonated -O- groups at 4-position of indole allowed for the availability of a great number of adsorption sites for MB molecules adsorption via electrostatic attraction. (iii) As MB is an

\begin{tabular}{|c|c|c|c|}
\hline Adsorbent & $q_{\mathrm{m}}\left(\mathrm{mg} \mathrm{g}^{-1}\right)$ & Temperature (K) & Ref. \\
\hline Amino-functionalised MOF & 762 & 303 & 35 \\
\hline Agar/grapheme oxide composite aerogel & 578 & 313 & 34 \\
\hline Walnut shell-based activated carbon & 315 & 298 & 36 \\
\hline Polydopamine microspheres & 98.82 & 318 & 29 \\
\hline $\begin{array}{l}\text { Core@double-shell structured magnetic halloysite nanotube nano- } \\
\text { hybrid }\end{array}$ & 714.29 & 318.15 & 8 \\
\hline 4-HIF aerogel & 1016.9 & 303 & This \\
\hline
\end{tabular}
ideally planar molecule with aromatic backbone and 4-HIF aerogel network also contain abundant aromatic rings, the $\pi-\pi$

Table 1 Comparison of the maximum adsorption values of adsorbents for MB from this study with other adsorbents in literature 


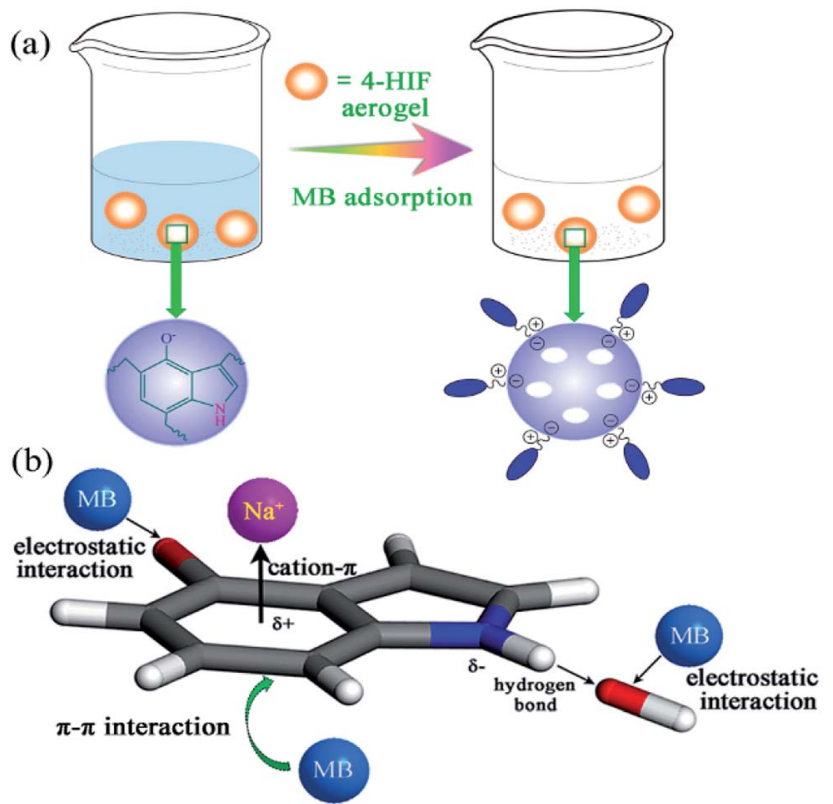

Fig. 5 (a) Schematic of adsorption process of MB. (b) Proposed mechanism for the removal of MB by 4-HIF aerogel.

interactions could occur between MB molecules and 4-HIF network. As shown in Fig. S7, $\uparrow$ the characteristic adsorption peaks of MB at 825,881 and $1591 \mathrm{~cm}^{-1}$ ascribed to the aromatic rings shift to 828,883 and $1596 \mathrm{~cm}^{-1}$, respectively. Density functional theory (DFT) ${ }^{40-43}$ calculations were performed and the result was shown in Fig. S8. $\dagger$ A correlation distance at 3.239 $\AA$ of MB to indole plane was obtained. This result also confirms the presence of $\pi-\pi$ interaction as each carbon atom in indole has a $\pi$ electron orbit which is perpendicular to the surface, and $\mathrm{MB}$ molecules containing $\mathrm{C}=\mathrm{C}$ double bonds and benzene rings with $\pi$ electrons could form $\pi-\pi$ bond with indole plane. ${ }^{37}$ In all, the synergistic effects of CIE, electrostatic interaction and $\pi-\pi$ interaction play a key role indicative of the efficient $\mathrm{MB}$ removal by 4 -HIF aerogel.

\section{Selectivity}

In order to investigate the selectivity of 4-HIF aerogel, we choose $\mathrm{MB}$ and methyl orange (MO) as model compounds for cationic dyes and anionic dye respectively. $40 \mathrm{~mL}$ dye mixture containing $10 \mathrm{mg} \mathrm{L}^{-1} \mathrm{MO}$ and $10 \mathrm{mg} \mathrm{L}^{-1} \mathrm{MB}$ at natural $\mathrm{pH}$ in the presence of $3 \mathrm{mg}$ 4-HIF aerogel was monitored by UV-vis spectrophotometer (Fig. 6a) at room temperature. After $60 \mathrm{~min}$, about $92.6 \%$ of MB was selectively separated with negligible MO being adsorbed. 4-HIF aerogel has a strong affinity for positively charged MB owing to the strong electrostatic interaction and particularly low affinity for negatively charged MO attributed to electrostatic repulsion. The results indicate 4-HIF aerogel has superior selectivity, suggesting the great potential for selective separation of cationic dyes.

\section{Application of 4-HIF aerogel to real water samples}

To assess the practical application of this material, we applied it for determination of in natural water samples. Common heavy
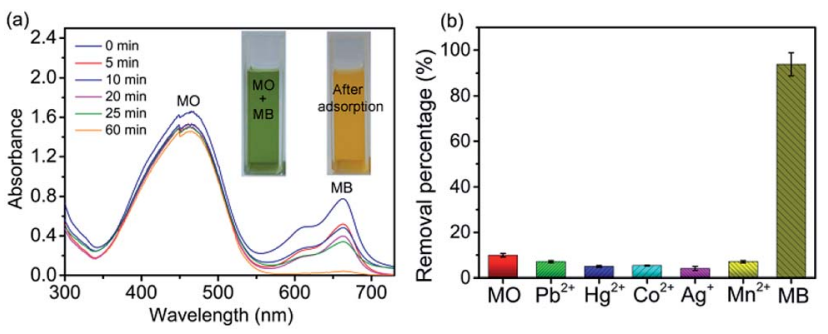

Fig. 6 (a) Temporal evolution of UV-vis spectra of dye mixture containing $\mathrm{MO}$ and $\mathrm{MB}$; (b) removal efficiency of 4-HIF aerogel towards $M B$ in real water samples in the presence of other common contaminant.

metals $\left(\mathrm{Pb}^{2+}, \mathrm{Ag}^{+}, \mathrm{Mn}^{2+}, \mathrm{Co}^{2+} \mathrm{Hg}^{2+}\right)$ and $\mathrm{MO}, \mathrm{MB}$ were spiked to 100 -fold diluted river water and the removal performance were evaluated. As can be seen from Fig. 6b, the removal percentage reaches up to $88.6 \%$ for $\mathrm{MB}$ while the negligible removal efficiency of other contaminants were observed. All these results suggested 4-HIF aerogel is qualified for practical application in integrative and efficient treatment of coexistent toxic pollutants.

\section{Desorption and regeneration}

For the desorption study, the saturated MB-loaded 4-HIF aerogel were easily regenerated by washing with ethanol and $0.1 \mathrm{M}$ $\mathrm{HCl}$. As can be seen in Fig. 7a, $0.1 \mathrm{M} \mathrm{HCl}$ was added to destroy the hydrogen bond between the indole amine and hydroxyl anion, the hydroxyl anions were neutralized and the electrostatic interaction was broken instantly. Subsequently, the desorbed MB molecules were easily extracted by ethanol from the 4HIF aerogel network. In addition, moderate heating at $50^{\circ} \mathrm{C}$ was also favorable for improving the regeneration efficiency. After desorption, $\mathrm{NaOH}$ treatment was performed to yield alkaline 4HIF aerogel for reuse. Five cycles of adsorption-desorption were completed and the results were presented in Fig. 7b. Obviously, the MB loaded 4-HIF aerogel could be efficiently regenerated by ethanol solutions with hydrochloric acid and 75\% capacity maintained after five cycles. All these results suggested the stability and reusability of 4-HIF aerogel in potential practical applications.

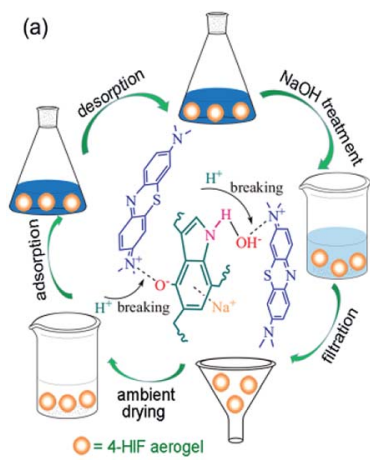

(b)

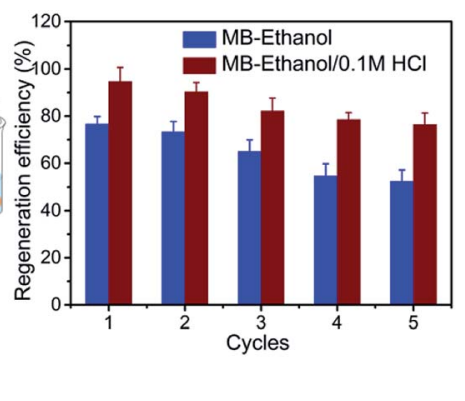

Fig. 7 (a) Mechanism for desorbing MB by 4-HIF aerogel and schematic of regeneration process. (b) Regeneration efficiency of 4-HIF aerogel for $\mathrm{MB}$ by ethanol, $0.1 \mathrm{M} \mathrm{HCl} /$ ethanol. 


\section{Conclusions}

In summary, a new type of indole-based 4-HIF aerogel has been rationally designed and successfully synthesized through a facile polymerization followed by ambient drying method. The SEM result show that the 4-HIF aerogel possesses highly crosslinked threedimensional porous network favorable for dye penetration and adsorption. The 4-HIF aerogels exhibit outstanding remediation performance towards $\mathrm{MB}$ from contaminated water based on the synergistic effects of CIE, electrostatic interaction and $\pi-\pi$ interaction. The adsorption of MB onto the 4-HIF aerogel was spontaneous and endothermic which possess high removal efficiency, fast adsorption kinetics, as well as ease of separation operation. Moreover, saturated 4-HIF aerogel can be regenerated easily by ethanol with $0.1 \mathrm{M} \mathrm{HCl}$ solution and maintained desirable recycling stability and reusability. We expect that this original research would lead to the development of advanced water purification techniques.

\section{Conflicts of interest}

There are no conflicts to declare.

\section{Acknowledgements}

This work was financially supported by the National Natural Science Foundation of China (21973076, 21504073, 21202134 and 11447215), the Sichuan Youth Science \& Technology Foundation (2016JQ0055), the Scientific Research Fund of Sichuan Provincial Education Department (16ZA0136 and 18ZA0495), the Longshan Academic Talent Research Supporting Program of SWUST (18LZX308 and 18LZX446).

\section{Notes and references}

1 V. Kampyli, D. A. S. Phillips and A. H. M. Renfrew, Dyes Pigm., 2004, 61, 165.

2 N. Peng, D. Hu, J. Zeng, Y. Li, L. Liang and C. Chang, ACS Sustainable Chem. Eng., 2016, 4, 7217.

3 R. W. Field, Nature, 2012, 489, 41.

4 J. Mathieu-Denoncourt, C. J. Martyniuk, S. R. de Solla, V. K. Balakrishnan and V. S. Langlois, Environ. Sci. Technol., 2014, 48, 2952.

5 P. Sharma, H. Kaur, M. Sharma and V. Sahore, Environ. Monit. Assess., 2011, 183, 151.

6 S. Zhang, X. Liu, M. Wang, B. Wu, B. Pan, H. Yang and H. Q. Yu, Environ. Sci. Technol. Lett., 2014, 1, 167.

7 Y. Zhan, X. Wan, S. He, Q. Yang and Y. He, Chem. Eng. J., 2018, 333, 132.

8 X. Wan, Y. Zhan, Z. Long, G. Zeng and Y. He, Chem. Eng. J., 2017, 330, 491.

9 J. Xiao, W. Lv, Z. Xie, Y. Tan, Y. Song and Q. Zheng, J. Mater. Chem. A, 2016, 4, 12126.

10 Y. Qin, L. Wang, C. Zhao, D. Chen, Y. Ma and W. Yang, ACS Appl. Mater. Interfaces, 2016, 8, 16690.

11 P. Hadi, J. Guo, J. Barford and G. McKay, Environ. Sci. Technol., 2016, 50, 5041.
12 Z. Wu, L. Zhang, Q. Guan, P. Ning and D. Ye, Chem. Eng. J., 2014, 258, 77.

13 F. Y. Yi, W. Zhu, S. Dang, J. P. Li, D. Wu, Y. H. Li and Z. M. Sun, Chem. Commun., 2015, 51, 3336.

14 G. Crini, Bioresour. Technol., 2006, 97, 1061.

15 C. A. Páez, M. S. Contreras, A. Léonard, S. Blacher, C. G. OliveraFuentes, J.-P. Pirard and N. Job, Adsorption, 2012, 18, 199.

16 R. Yu, Y. Shi, D. Yang, Y. Liu, J. Qu and Z.-Z. Yu, ACS Appl. Mater. Interfaces, 2017, 9, 21809.

17 J. C. Ma and D. A. Dougherty, Chem. Rev., 1997, 97, 1303.

18 D. A. Dougherty, Acc. Chem. Res., 2013, 46, 885.

19 C. R. Kennedy, S. Lin and E. N. Jacobsen, Angew. Chem., Int. Ed., 2016, 55, 12596.

20 A. J. Neel, M. J. Hilton, M. S. Sigman and F. D. Toste, Nature, 2017, 543, 637.

21 M. Kah, G. Sigmund, F. Xiao and T. Hofmann, Water Res., 2017, 124, 673.

22 A. S. Reddy and G. N. Sastry, J. Phys. Chem. A, 2005, 109, 8893.

23 W. Wei, G. Chang, Y. Xu and L. Yang, J. Mater. Chem. A, 2018, 6, 18794.

24 P. Yang, L. Yang, Y. Wang, L. Song, J. Yang and G. Chang, J. Mater. Chem. A, 2019, 7, 531.

25 G. Chang, Z. Shang, T. Yu and L. Yang, J. Mater. Chem. A, 2016, 4, 2517.

26 G. Chang, L. Yang, J. Yang, M. P. Stoykovich, D. Xu, J. Cui and D. Wang, Adv. Mater., 2018, 30, 1704234.

27 L. J. Juszczak and A. S. Eisenberg, J. Am. Chem. Soc., 2017, 139, 8302.

28 A. Schlüter, K. Biebe and W. S. Sheldrick, Inorg. Chim. Acta, 2002, 340, 35.

29 J. Fu, Z. Chen, M. Wang, S. Liu, J. Zhang, J. Zhang, R. Han and Q. Xu, Chem. Eng. J., 2015, 259, 53.

30 S. S. Elanchezhiyan and S. Meenakshi, J. Cleaner Prod., 2016, 139, 1339.

31 S. S. D. Elanchezhiyan, S. M. Prabhu and S. Meenakshi, Int. J. Biol. Macromol., 2018, 112, 294.

32 S. Hong, C. F. Schaber, K. Dening, E. Appel, S. N. Gorb and H. Lee, Adv. Mater., 2014, 26, 7581.

33 C. Liang, G. Sha and S. Guo, J. Non-Cryst. Solids, 2000, 271, 167.

34 L. Chen, Y. Li, Q. Du, Z. Wang, Y. Xia, E. Yedinak, J. Lou and L. Ci, Carbohydr. Polym., 2017, 155, 345.

35 E. Haque, V. Lo, A. I. Minett, A. T. Harris and T. L. Church, J. Mater. Chem. A, 2014, 2, 193.

36 J. Yang and K. Qiu, Chem. Eng. J., 2010, 165, 209.

37 Z. Wu, H. Zhong, X. Yuan, H. Wang, L. Wang, X. Chen, G. Zeng and Y. Wu, Water Res., 2014, 67, 330.

38 F. Zhao, E. Repo, D. Yin, Y. Meng, S. Jafari and M. Sillanpää, Environ. Sci. Technol., 2015, 49, 10570.

39 R. Kurrey, M. Mahilang, M. K. Deb, J. Nirmalkar, K. Shrivas, S. Pervez, M. K. Rai and J. Rai, Food Chem., 2019, 270, 459.

40 L. Yang, C. Wang, G. Chang and X. Ren, Sens. Actuators, B, 2017, 240, 212.

41 P. Yang, L. Yang, J. Yang, X. Luo and G. Chang, High Perform. Polym., 2019, 31, 238.

42 Y. Li, L. Yang, M. Du and G. Chang, Analyst, 2019, 144, 1260. 43 S. Gao, G. Shi and H. Fang, Nanoscale, 2016, 8, 1451. 